PROCEEDINGS OF THE

AMERICAN MATHEMATICAL SOCIETY

Volume 127, Number 12, Pages 3601-3603

S 0002-9939(99)04937-0

Article electronically published on May 13, 1999

\title{
CHAOTIC POLYNOMIALS ON FRÉCHET SPACES
}

\author{
ALFREDO PERIS
}

(Communicated by Theodore W. Gamelin)

\begin{abstract}
Contrary to the case of polynomials on Banach spaces, in which it is known that no hypercyclic homogeneous polynomial of degree $m \geq 2$ exists on any Banach space, we construct for each $m \geq 2$ a chaotic $m$-homogeneous polynomial $P$ on the Fréchet space $\mathcal{H}(\mathbb{C})$.
\end{abstract}

A map $T: X \rightarrow X$ on a metric space $X$ is chaotic (see [3]) if (a) $T$ is transitive (which for complete separable $X$ is equivalent to the existence of $x \in X$ whose orbit $\operatorname{Orb}(T, x):=\left\{x, T x, T^{2} x, \ldots\right\}$ is dense in $\left.X\right)$, (b) the periodic points of $T$ are dense in $X$, and (c) $T$ has sensitive dependence on initial conditions. Banks et al. [1] showed that (c) is redundant in the definition of Devaney.

We present an example of a chaotic $m$-homogeneous continuous polynomial $P: \mathcal{H}(\mathbb{C}) \rightarrow \mathcal{H}(\mathbb{C})$. This must be compared with a result of Bernardes [2], who showed that for $m>1$ there are no continuous $m$-homogeneous polynomials admitting a vector with dense orbit (hypercyclic in the usual terminology) on any Banach space. The result of Bernardes is a consequence of the inequality $\left\|P^{n} x\right\| \leq$ $\|P\|^{1+m+\ldots+m^{n-1}}\|x\|^{m^{n}}$ for every $x \in X$ if $P$ is a continuous $m$-homogeneous polynomial $(m>1)$ on a Banach space $X$, since no hypercyclic vector for $P$ could lie on the ball centered at 0 of radius $r:=1 /\|P\|$. This implies that $P$ does not admit any hypercyclic vector.

On $\mathcal{H}(\mathbb{C})$ we consider the increasing sequence of norms $\left(\|\cdot\|_{k}\right)_{k}$ defined by

$$
\|f\|_{k}:=\sup _{j \geq 0} \frac{\left|f^{(j)}(0)\right|}{j !} k^{j}, \quad k \in \mathbb{N}, \quad f \in \mathcal{H}(\mathbb{C}),
$$

which define the natural Fréchet topology on $\mathcal{H}(\mathbb{C})$.

Our notation is standard. We refer to the monograph [6] for Fréchet spaces and to [4] for polynomials on locally convex spaces.

Theorem 1. For each $m$ natural $(m \geq 2)$ there exists a chaotic $m$-homogeneous polynomial $P: \mathcal{H}(\mathbb{C}) \rightarrow \mathcal{H}(\mathbb{C})$.

Proof. Let us define $P: \mathcal{H}(\mathbb{C}) \rightarrow \mathcal{H}(\mathbb{C})$ by $(P f)(z):=\sum_{j \geq 0} \frac{\left(f^{(j+1)}(0)\right)^{m}}{j !} z^{j}$ for every $f \in \mathcal{H}(\mathbb{C})$ and for every $z \in \mathbb{C} . \quad P$ is obviously a well-defined $m$-homogeneous polynomial. We first prove that $P$ is hypercyclic.

Received by the editors December 29, 1997 and, in revised form, February 17, 1998.

1991 Mathematics Subject Classification. Primary 46G20, 46A04, 58F08.

This research was supported in part by DGICYT under Proyecto PB94-0541.

(C)1999 American Mathematical Society 
Indeed, by the hypercyclicity of the derivative operator $D: \mathcal{H}(\mathbb{C}) \rightarrow \mathcal{H}(\mathbb{C})$ (see $[5])$, there exists $x \in \mathcal{H}(\mathbb{C})$, a hypercyclic vector for $D$. We denote by $x_{j}:=x^{(j)}(0)$, $j \in \mathbb{N}$, and pick an increasing sequence $\left(n_{k}\right)_{k}$ of natural numbers such that $\left(D^{n_{k}} x\right)_{k}$ is dense in $\mathcal{H}(\mathbb{C})$ and

$$
\begin{aligned}
& \text { (i) }\left\|\sum_{j \geq n_{k+1}-n_{k}} \frac{x_{j+n_{k}}}{j !} z^{j}\right\|_{k}<\frac{1}{k}, \\
& \text { (ii) } \sup \left\{\frac{k^{j}}{j !} / j \geq n_{k+1}-n_{k}\right\}<\frac{1}{k},
\end{aligned}
$$

for each $k \in \mathbb{N}$.

Now we select $\left(y_{j}\right)_{j \geq 0}$ such that

$$
y_{j}^{m^{n_{k}}}=x_{j}, \quad n_{k} \leq j<n_{k+1} \quad\left(n_{0}:=0\right) \quad \forall k \in \mathbb{N} .
$$

Then

$$
y:=y(z):=\sum_{j \geq 0} \frac{y_{j}}{j !} z^{j} \in \mathcal{H}(\mathbb{C})
$$

since $\left|y_{j}\right| \leq 1+\left|x_{j}\right|, j \geq 0$. Moreover, the selection of $\left(y_{j}\right)_{j \geq 0}$ and the definition of $P$ imply

(iii) $\left(P^{n_{k}} y\right)^{(j)}(0)=\left(D^{n_{k}} x\right)^{(j)}(0), 0 \leq j<n_{k+1}-n_{k}, \forall k \in \mathbb{N}$.

Given $n \in \mathbb{N}, f \in \mathcal{H}(\mathbb{C})$ and $\varepsilon>0$, we fix $k>n$ such that $1 / k<\varepsilon / 4$ and $\left\|D^{n_{k}} x-f\right\|_{n}<\varepsilon / 4$. Then, from (i), (ii) and (iii), we get

$$
\begin{gathered}
\left\|P^{n_{k}} y-f\right\|_{n} \\
\leq\left\|P^{n_{k}} y-\sum_{n_{k+1}-n_{k}>j \geq 0} \frac{x_{j+n_{k}}}{j !} z^{j}\right\|_{n}+\left\|\sum_{j \geq n_{k+1}-n_{k}} \frac{x_{j+n_{k}}}{j !} z^{j}\right\|_{n}+\left\|D^{n_{k}} x-f\right\|_{n} \\
<\left\|P^{n_{k}} y-\sum_{n_{k+1}-n_{k}>j \geq 0} \frac{\left(D^{n_{k}} x\right)^{(j)}(0)}{j !} z^{j}\right\|_{k}+\frac{1}{k}+\frac{\varepsilon}{4} \\
<\sup \left\{\frac{\left|y_{j+n_{k}}\right|^{m^{n_{k}}}}{j !} k^{j} / j \geq n_{k+1}-n_{k}\right\}+\frac{\varepsilon}{2} \\
\leq \sup \left\{\frac{1+\left|x_{j+n_{k}}\right|}{j !} k^{j} / j \geq n_{k+1}-n_{k}\right\}+\frac{\varepsilon}{2} \\
<\sup \left\{\frac{\left|x_{j+n_{k} \mid}\right|}{j !} k^{j} / j \geq n_{k+1}-n_{k}\right\}+\frac{1}{k}+\frac{\varepsilon}{2}<\varepsilon .
\end{gathered}
$$

This implies that $P$ is hypercyclic. To conclude the proof we show that the periodic vectors of $P$ are dense in $\mathcal{H}(\mathbb{C})$. We are done if, given $m \in \mathbb{N}$ and an arbitrary polynomial $p(z)=\sum_{j=1}^{n} \frac{\alpha_{j}}{j !} z^{j}$, we find $u \in \mathcal{H}(\mathbb{C})$ periodic for $P$ such that $\|u-p\|_{m}<$ $1 / m$. To do this, take $k>n$ satisfying

$$
\text { (iv) } \sup \left\{\frac{M}{j !} m^{j} \quad / \quad j \geq k\right\}<\frac{1}{m}
$$

where $M:=\sup \left\{1+\left|\alpha_{j}\right| / \quad 1 \leq j \leq n\right\}$. Choose $\left(u_{j}\right)_{j \geq 0}$ such that

$$
u_{j}:=\alpha_{j} \quad\left(\alpha_{j}:=0 \quad \text { if } j>n\right), \quad u_{j+r k}^{m^{k}}=u_{j+(r-1) k} \quad \text { if } 0 \leq j<k \quad \text { and } r \geq 1 .
$$


We have that $u(z):=\sum_{j>0} \frac{u_{j}}{j !} z^{j} \in \mathcal{H}(\mathbb{C})$, since $\left|u_{j}\right| \leq 1+\left|\alpha_{j}\right|, j \geq 0$. By the selection of $\left(u_{j}\right)_{j \geq 0}$ and (iv) we also get that $u$ is $k$-periodic for $P$ and

$$
\|u-p\|_{m}=\left\|\sum_{j \geq k} \frac{u_{j}}{j !} z^{j}\right\|_{m} \leq \sup \left\{\frac{M}{j !} m^{j} \quad / \quad j \geq k\right\}<\frac{1}{m} .
$$

We conclude that $P$ is chaotic.

\section{REFERENCES}

[1] J. Banks, J. Brooks, G. Cairns, G. Davis, P. Stacey, On Devaney's definition of chaos, Amer. Math. Monthly 99 (1992), 332-334. MR 93d:54059

[2] N. C. Bernardes, On orbits of polynomial maps in Banach spaces, Quaestiones Math. (to appear).

[3] R. L. Devaney, An Introduction to Chaotic Dynamical Systems, Addison-Wesley, 1989. MR 91a:58114

[4] S. Dineen, Complex Analysis in Locally Convex Spaces, North-Holland, Amsterdam-New York-Oxford, 1981. MR 84b:46050

[5] G. R. MacLane, Sequences of derivatives on normal families, J. Analyse Math. 2 (1952), 72-87. MR 14:741d

[6] R. Meise, D. Vogt, Introduction to Functional Analysis, Oxford Univ. Press, 1997. CMP 98:04

Departamento de Matemática Aplicada, E.T.S. Arquitectura, Universidad Politécnica de Valencia, E-46071 Valencia, Spain

E-mail address: aperis@pleiades.upv.es 